\title{
Differential diffusion effects inclusion with flamelet generated manifold for the modeling of stratified premixed cooled flames
}

\section{Citation for published version (APA):}

Donini, A., Bastiaans, R. J. M., Oijen, van, J. A., \& Goey, de, L. P. H. (2015). Differential diffusion effects inclusion with flamelet generated manifold for the modeling of stratified premixed cooled flames. Proceedings of the Combustion Institute, 35(1), 831-837. https://doi.org/10.1016/j.proci.2014.06.050

\section{Document license:}

TAVERNE

DOI:

10.1016/j.proci.2014.06.050

Document status and date:

Published: 01/01/2015

\section{Document Version:}

Publisher's PDF, also known as Version of Record (includes final page, issue and volume numbers)

\section{Please check the document version of this publication:}

- A submitted manuscript is the version of the article upon submission and before peer-review. There can be important differences between the submitted version and the official published version of record. People interested in the research are advised to contact the author for the final version of the publication, or visit the $\mathrm{DOI}$ to the publisher's website.

- The final author version and the galley proof are versions of the publication after peer review.

- The final published version features the final layout of the paper including the volume, issue and page numbers.

Link to publication

\section{General rights}

Copyright and moral rights for the publications made accessible in the public portal are retained by the authors and/or other copyright owners and it is a condition of accessing publications that users recognise and abide by the legal requirements associated with these rights.

- Users may download and print one copy of any publication from the public portal for the purpose of private study or research.

- You may not further distribute the material or use it for any profit-making activity or commercial gain

- You may freely distribute the URL identifying the publication in the public portal.

If the publication is distributed under the terms of Article 25fa of the Dutch Copyright Act, indicated by the "Taverne" license above, please follow below link for the End User Agreement:

www.tue.nl/taverne

Take down policy

If you believe that this document breaches copyright please contact us at:

openaccess@tue.nl

providing details and we will investigate your claim. 


\title{
Differential diffusion effects inclusion with flamelet generated manifold for the modeling of stratified premixed cooled flames
}

\author{
A. Donini* , R.J.M. Bastiaans, J.A. van Oijen, L.P.H. de Goey \\ Combustion Technology Group, Department of Mechanical Engineering, Eindhoven University of Technology, Den \\ Dolech 2, 5612 AZ Eindhoven, Netherlands
}

Available online 10 July 2014

\begin{abstract}
CFD predictions of flame position, stability and emissions are essential in order to obtain optimized combustor designs in a cost efficient way. However, the numerical modeling of practical combustion systems is a very challenging task. As a matter of fact, the use of detailed reaction mechanisms is necessary for reliable predictions, especially for highly diffusive fuels. Unfortunately, the modeling of the full detail of practical combustion equipment is currently prohibited by the limitations in computing power, given the large number of species and reactions involved. The Flamelet Generated Manifold (FGM) method reduces these computational costs by several orders of magnitude without loosing too much accuracy. Hereby, FGM enables the application of reliable chemistry mechanisms in CFD simulations of combustion processes. In the FGM technique the progress of the flame is generally described by a few control variables. For each control variable a transport equation is solved during run-time. The flamelet system is computed in a pre-processing stage, and a manifold with all the information about combustion is stored in a tabulated form. In the present paper, the FGM model is implemented for the analysis of partially premixed non-adiabatic flames, including the effects of differential diffusion. Subsequently, a computational analysis of partially premixed non-adiabatic flames is presented. In this scope, a series of test simulations is performed using FGM for a two dimensional geometry, characterized by a distinctive stratified methane/ air inlet, and compared with detailed chemistry simulations. The results indicate that detailed simulations are well reproduced with the FGM technique.
\end{abstract}

(C) 2014 The Combustion Institute. Published by Elsevier Inc. All rights reserved.

Keywords: Differential diffusion; Flamelet generated manifolds; Heat loss; Mixture fraction; Premixed

\section{Introduction}

In industry the development of clean and efficient technologies for the combustion process is

\footnotetext{
* Corresponding author.

E-mail address: andrea.donini@gmail.com
}

achieved by a combination of experimental and numerical research. Experimental testing is in general extremely expensive, and a great reduction of the costs could be made by maximizing the usage of simulations in the design phase. Unfortunately, the modeling of the full detail of practical combustion equipment is currently prohibited by the limitations in computing power, given the large

(A. Donini). 
number of species and reactions involved. The Flamelet-Generated Manifold (FGM) [1] method, also referred to as Flamelet Prolongation of ILDM [2], reduces these computational costs by several orders of magnitude without loosing too much accuracy. Hereby FGM enables the application of reliable chemistry mechanisms in CFD simulations of combustion processes. The approach is based on the idea that the most important aspects of the internal structure of the flame fronts should be taken into account. In the FGM technique the course of the reaction is defined in terms of a few control variables, for which transport equations are solved during runtime. And here lies one of the main strengths of the FGM technique, which is that the number of independent control variables can be increased for a better description of the combustion phenomena. This means that, starting with a single reaction progress variable, the accuracy of the method can be straightforwardly extended for the inclusion of heat loss, mixture fraction and differential diffusive effects, as shown in the following section. The flamelet system is computed in a pre-processing stage, and a manifold with all the information about combustion is stored in a tabulated form. To this purpose a laminar flamelet database is generated from a one-dimensional flamelet calculation performed with full kinetics and detailed transport. The FGM technique has proven to be very accurate for laminar premixed Bunsen flames including heat loss effects [1], highly stretched premixed counter-flow flames [3] and confined triple flames [4]. This technique performed well also in DNS of a turbulent expanding flame [5], showing that a single control variable can give accurate predictions on the local mass burning rate. Recently the approach has proven to be appropriate also for the computation of turbulent partially premixed flames [6,7], also with heat loss [8]. A large part of current combustion models adopt a unity Lewis number assumption, imposing that mass and heat diffuse at an equal rate in the flame. On the opposite, when $L e$ is not equal to one, species and heat locally redistribute. This phenomenon is also referred to as differential diffusion [9]. The inclusion of this effect is important especially in the case of laminar flames. With the purpose of including every aspect in the numerical modeling of differentially diffusive flames, in this paper the FGM model is extended for differential diffusion inclusion and applied to cooled stratified premixed flames by means of a 3D manifold where the control variables are represented by the progress variable $\mathcal{Y}$, enthalpy $h$ and mixture fraction $Z$. The combined inclusion of these effects represents a challenge. At the best of the authors' knowledge, the application of FGM in combination with heat loss, mixture fraction variations and differential diffusion effects has never been studied in literature. Such implementation of the FGM technique is therefore applied for two-dimensional test case flame simulations with stratified inlet and accurately compared with detailed chemistry computations. The results of the present validation are supported and further motivated by the detailed Le $=1$ comparison given in [10] for the same geometry and conditions. The fuel adopted for the simulations is methane, because of its mild diffusivity. In fact, the work presented in this paper represents a test and validation of the method, as a first and necessary step before considering highly diffusive fuels such as hydrogen.

\section{The FGM database generation}

In the FGM technique the whole chemistry is directly retrieved from a laminar flamelet database generated from multiple one-dimensional flamelet calculations performed with detailed kinetics and transport. In this study cooled stratified premixed methane/air combustion is considered, at atmospheric pressure conditions. The FGM consists of a 3D manifold in which the control variables are represented by the progress variable $\mathcal{Y}$, enthalpy $h$ and mixture fraction $Z$. The minimal dimension of the manifold in the phase space is determined by an accuracy assessment [11], however several automated techniques have been recently proposed, e.g. [12,13]. As described in the introduction, differential diffusion results in local changes in mass fraction and enthalpy. In order to accurately predict such effect, mass fraction and enthalpy must be included as control variable of the FGM description. The progress variable $\mathcal{Y}$ is defined as a linear combination of species mass fraction: $\mathcal{Y}=\sum_{i=1}^{N_{s}} \alpha_{i} Y_{i}$, where $Y_{i}$ is the mass fraction of specie $i$ and $N_{s}$ the total number of species. The weighting coefficients $\alpha_{i}$ are arbitrarily chosen, with the only restriction of ensuring a monotonic profile of $\mathcal{Y}$ in the whole interval between the unburned mixture and the chemical equilibrium. For the calculations described in this paper, it is chosen: $\alpha_{\mathrm{CO}_{2}}=$ $M_{\mathrm{CO}_{2}}^{-1}, \alpha_{\mathrm{H}_{2}}=M_{\mathrm{H}_{2}}^{-1}, \alpha_{\mathrm{H}_{2} \mathrm{O}}=M_{\mathrm{H}_{2} \mathrm{O}}^{-1}, \alpha_{\mathrm{O}_{2}}=M_{\mathrm{O}_{2}}^{-1}, \alpha_{i}=0$ $\forall i \notin\left\{\mathrm{CO}_{2}, \mathrm{H}_{2}, \mathrm{H}_{2} \mathrm{O}, \mathrm{O}_{2}\right\}$, in which $\mathrm{M}_{i}$ is the molar mass of element $i$. Heat loss to the combustion chamber walls cause enthalpy not to be conserved throughout the domain. In order to take this into account in the tabulation process, the laminar flamelets have to be solved for different values of enthalpy, introducing enthalpy $h$ as a control variable. The flamelets are computed as steady, fully premixed, flat flames, for a given pressure, composition and temperature of the inlet mixture. At the burned side (chemical equilibrium) Neumann type boundary conditions are imposed instead. The procedure for the creation of enthalpy-decreasing set of flamelets might be 
performed in different ways, but the most straightforward are: (1) decreasing the enthalpy of free adiabatic flamelets by simply diminishing step by step the inlet temperature (therefore $h_{-\infty}$ ), (2) calculation of burner-stabilized flamelets, by imposing a certain amount (step by step increasing) of heat loss to the burner. Here both methods are used in order to have a fairly complete manifold. In fact burner-stabilized flamelets allow to impose very high values of cooling. It has been proven that the choice of the enthalpy-decrease method for the tabulation procedure has negligible influence on the final result [1]. In partially premixed flames fuel and oxidizer are not perfectly mixed, therefore variations in local element composition occur. This effect can be described by the mixture fraction $Z$. Mixture fraction is expressed in terms of element mass fractions $Z_{j}$ of element $j$ [14]:

$Z=\frac{2 M_{H}^{-1}\left(Z_{H}-Z_{H, 2}\right)+0.5 M_{C}^{-1}\left(Z_{C}-Z_{C, 2}\right)-M_{O}^{-1}\left(Z_{O}-Z_{O, 2}\right)}{2 M_{H}^{-1}\left(Z_{H, 1}-Z_{H, 2}\right)+0.5 M_{C}^{-1}\left(Z_{C, 1}-Z_{C, 2}\right)-M_{O}^{-1}\left(Z_{O, 1}-Z_{O, 2}\right)}$,

where the subscripts 1 and 2 denote respectively pure fuel and pure oxidizer. $Z$ is defined as a linear combination of element mass fractions, and it is therefore conserved throughout the chemical reaction. In order to take this into account in the tabulation process, the laminar flamelets have to be solved for different values of mixture fraction, introducing $Z$ as a control variable. The convenience of this approach is demonstrated in previous studies on partially premixed flames, e.g. $[6,15]$. A graphical representation of the mixture fraction along the flamelets obtained with this procedure is shown in Fig. 1. In this figure the mixture fraction is not constant along the flamelets, as a direct result of differential diffusion. The flamelet equations are solved with a specialized 1D flame code [16]), coupled with the GRIMech 3.0 reaction mechanism [17] which consists of 325 elementary reactions between 53 species with hydrocarbons up to propane.

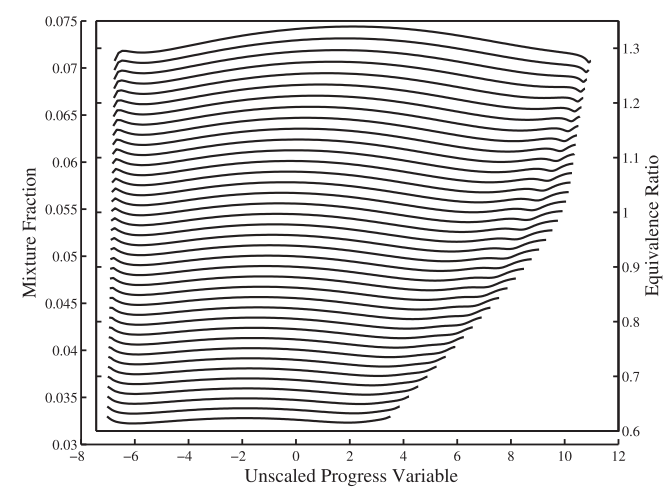

Fig. 1. Mixture fraction along the flamelets composing the manifold, for $h=h_{\max }$.
Flamelet solutions are mapped in such a way that any thermochemical variable $\psi$ is directly described as $\psi=\psi(\mathcal{Y}, h, Z)$. The tabulation procedure consists in storing all the thermochemical variables $\psi$ in a tabulated form, on a defined tridimensional grid for which is divided the $\mathcal{Y}-h-Z$ range. The number of points adopted for the manifold created is $n_{p v}=250$ in the progress variable dimension, $n_{h}=40$ for the enthalpy and $n_{Z}=86$ for the mixture fraction. The calculation of transport and thermodynamic coefficients is performed by means of a mixture averaged approach during the pre-processing stage, and stored in the manifold together with the chemical data in a tabulated form (although several storage methods are available, e.g. $[18,19])$. An overview of the resulting manifold can be seen in Fig. 2, where various scalars $\psi$ are represented as a function of the progress variable and equivalence ratio for the highest enthalpy value. The highest enthalpy levels essentially corresponds to the adiabatic flame conditions. The equivalence ratio is chosen instead of $Z$ for the sake of clarity in the plots.

\section{Implementation of differential diffusion effects in FGM}

The control variable equations describing thermo-diffusive unstable low Lewis number flames are directly following from the transport equations of $\mathcal{Y}, h$ and $Z$, formerly derived (for a different application) in [11]. However, in [11] only 2 control variables were used, and stretch was applied to the flamelets. These equations, applied to the present $3-\mathrm{D}$ case read:

$$
\begin{aligned}
& \frac{\partial(\rho \mathcal{Y})}{\partial t}+\nabla \cdot(\rho \mathbf{u} \mathcal{Y})-\nabla \cdot\left(\frac{\lambda}{c_{p}} \nabla \mathcal{Y}\right) \\
& =\nabla \cdot\left(\frac{\lambda}{c_{p}} \sum_{i=1}^{N s} \alpha_{i}\left(\frac{1}{L e_{i}}-1\right) \nabla \mathcal{Y}\right)+\dot{\omega}_{\mathcal{Y}} \\
& \frac{\partial(\rho h)}{\partial t}+\nabla \cdot(\rho \mathbf{u} h)-\nabla \cdot\left(\frac{\lambda}{c_{p}} \nabla h\right) \\
& =\nabla \cdot\left(\frac{\lambda}{c_{p}} \sum_{i=1}^{N s} h_{i}\left(\frac{1}{L e_{i}}-1\right) \nabla Y_{i}\right) \\
& \frac{\partial(\rho Z)}{\partial t}+\nabla \cdot(\rho \mathbf{u} Z)-\nabla \cdot\left(\frac{\lambda}{c_{p}} \nabla Z\right) \\
& =\nabla \cdot\left(\frac{\lambda}{c_{p}} \sum_{i=1}^{N s} \zeta_{i}\left(\frac{1}{L e_{i}}-1\right) \nabla Y_{i}\right)
\end{aligned}
$$

in which $\rho$ is the mass density, $\mathbf{u}$ the velocity, $\lambda$ the thermal conductivity of the mixture, $c_{p}$ the specific heat at constant pressure and $\dot{\omega}_{y}$ the progress variable source term. The first term of the right hand 
side of these equation is the differential diffusion term. This term has the same structure for all three control variable equations, and it differs in the coefficients only. Thus, a model for this term can be first derived for the progress variable equation, and then generalized for any control variable. Given the FGM assumption $Y_{i}=Y_{i}(\mathcal{Y}, h, Z)$, applying the chain rule the differential diffusion term, and assuming that locally the controlling variables $(\mathcal{Y}, h, Z)$ are as a function of $\mathcal{Y}$ solely $(h, Z)=\left(h^{1 D}, Z^{1 D}\right) \quad[11]$, the progress variable equation may be recast in

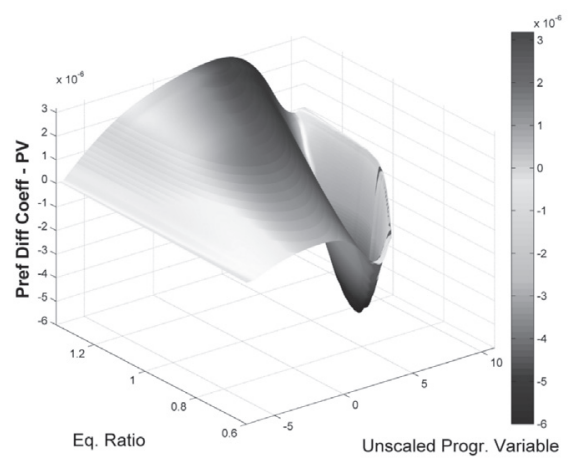

(a)

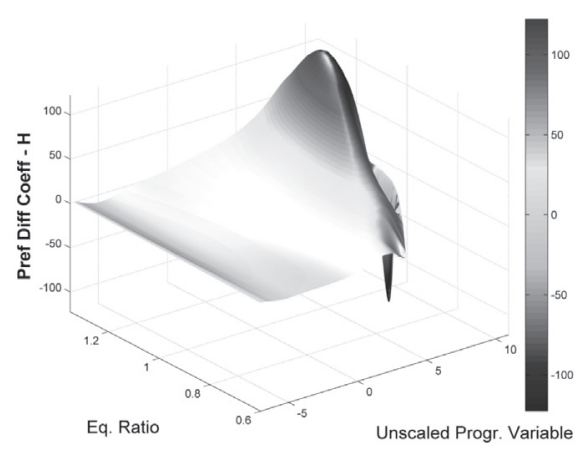

(b)

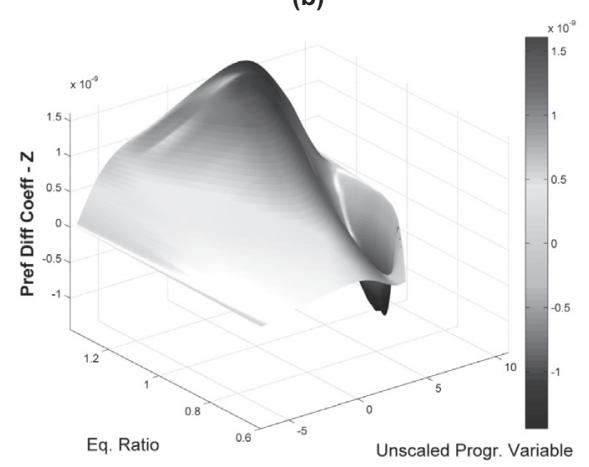

(c)

Fig. 2. Representations of the 3D manifold at the highest enthalpy level. Differential diffusion coefficient profile along the manifold: (a) $d_{\mathcal{C}_{1}}$, (b) $d_{\mathcal{C}_{2}}$, (c) $d_{\mathcal{C}_{3}}$.

$$
\begin{aligned}
& \frac{\partial(\rho \mathcal{Y})}{\partial t}+\nabla \cdot(\rho \mathbf{u} \mathcal{Y})-\nabla \cdot\left(\frac{\lambda}{c_{p}} \nabla \mathcal{Y}\right) \\
& =\nabla \cdot\left(d_{\mathcal{Y}} \nabla \mathcal{Y}\right)+\dot{\omega}_{\mathcal{Y}},
\end{aligned}
$$

with

$$
d_{\mathcal{Y}}=\frac{\lambda}{c_{p}} \sum_{i=1}^{N_{s}}\left(\frac{1}{L e_{i}}-1\right) \alpha_{i}\left(\frac{\partial Y_{i}}{\partial \mathcal{Y}}+\frac{\partial Y_{i}}{\partial h} \frac{\partial h^{1 D}}{\partial \mathcal{Y}}+\frac{\partial Y_{i}}{\partial Z} \frac{\partial Z^{1 D}}{\partial \mathcal{Y}}\right)
$$

The coefficient $d_{y}$ is very convenient because it can be calculated in the pre-processing stage and stored in the manifold together with the other thermochemical variables. The generic control variable can be defined as $\mathcal{C}_{k}=\left\{\mathcal{C}_{1}, \mathcal{C}_{2}, \ldots, \mathcal{C}_{\mathrm{N}_{\mathrm{CV}}}\right\}$ with $k \in\left[1, N_{C V}\right]$, where $N_{C V}$ is the number of control variables. For our case it is clear that with this definition it results $N_{C V}=3$ and $\mathcal{C}_{1}=\mathcal{Y}, \mathcal{C}_{2}=h$ and $\mathcal{C}_{3}=Z$. Generalizing the derivation of Eq. (5), the control variable conservation equation reads:

$$
\begin{aligned}
& \frac{\partial\left(\rho \mathcal{C}_{k}\right)}{\partial t}+\nabla \cdot\left(\rho \mathbf{u} \mathcal{C}_{k}\right)-\nabla \cdot\left(\frac{\lambda}{c_{p}} \nabla \mathcal{C}_{k}\right) \\
& \quad=\nabla \cdot\left(d_{\mathcal{C}_{k}} \nabla \mathcal{Y}\right)+\dot{\omega}_{\mathcal{C}_{k}},
\end{aligned}
$$

in which $d_{\mathcal{C}_{k}}$ represents the differential diffusion coefficient for the control variable $\mathcal{C}_{k}$ :

$d_{\mathcal{C}_{k}}=\frac{\lambda}{c_{p}} \sum_{i=1}^{N_{s}}\left[\left(\frac{1}{L e_{i}}-1\right) \mathcal{D}_{k, i} \sum_{i=1}^{N_{C V}} \frac{\partial Y_{i}}{\partial \mathcal{C}_{k}} \frac{\partial \mathcal{C}_{k}^{1 D}}{\partial \mathcal{C}_{1}}\right]$

The species diffusion coefficient value $\mathcal{D}_{k, i}$ is depending on the control variable, as described in Table 1. In Eq. (7) the term $\dot{\omega}_{\mathcal{C}_{k}}$ is the chemical source, and it is non-zero only for the progress variable transport, in which $\dot{\omega}_{\mathcal{C}_{1}}=\dot{\omega}_{\mathcal{Y}}$. The differential diffusion coefficients for the control variables of our case are shown in Table 2.

During run-time the FGM is linked to a standard CFD code. Together with momentum and continuity equations, the CFD code must solve the conservation equation for the progress

Table 1

Species diffusion coefficient for the controlling variables.

\begin{tabular}{ll}
\hline $\mathcal{C}_{k}$ & Species diffusion coefficient $\mathcal{D}_{k, i}$ \\
\hline $\mathcal{C}_{1}=\mathcal{Y}$ & $\mathcal{D}_{1, i}=\alpha_{i}$ \\
$\mathcal{C}_{2}=h$ & $\mathcal{D}_{2, i}=h_{i}$ \\
$\mathcal{C}_{3}=Z$ & $\mathcal{D}_{3, i}=\zeta_{i}$ \\
\hline
\end{tabular}

Table 2

Preferential diffusion coefficients for the controlling variables.

\begin{tabular}{l}
$\overline{\text { Differential diffusion coefficient } d_{\mathcal{C}_{k}}}$ \\
$d_{\mathcal{C}_{1}}=\frac{\lambda}{c_{p}} \sum_{i=1}^{N_{s}}\left(\frac{1}{L e_{i}}-1\right) \alpha_{i}\left(\frac{\partial Y_{i}}{\partial \mathcal{C}_{1}}+\frac{\partial Y_{i}}{\partial \mathcal{C}_{2}} \frac{\partial \mathcal{C}_{2}^{1 D}}{\partial \mathcal{C}_{1}}+\frac{\partial Y_{i}}{\partial \mathcal{C}_{3}} \frac{\partial \mathcal{C}_{3}^{1 D}}{\partial \mathcal{C}_{1}}\right)$ \\
$d_{\mathcal{C}_{2}}=\frac{\lambda}{c_{p}} \sum_{i=1}^{N_{s}}\left(\frac{1}{L e_{i}}-1\right) h_{i}\left(\frac{\partial Y_{i}}{\partial \mathcal{C}_{1}}+\frac{\partial Y_{i}}{\partial \mathcal{C}_{2}} \frac{\partial \mathcal{C}_{2}^{1 D}}{\partial \mathcal{C}_{1}}+\frac{\partial Y_{i}}{\partial \mathcal{C}_{3}} \frac{\partial \mathcal{C}_{3}^{1 D}}{\partial \mathcal{C}_{1}}\right)$ \\
$d_{\mathcal{C}_{3}}=\frac{\lambda}{c_{p}} \sum_{i=1}^{N_{s}}\left(\frac{1}{L e_{i}}-1\right) \zeta_{i}\left(\frac{\partial Y_{i}}{\partial \mathcal{C}_{1}}+\frac{\partial Y_{i}}{\partial \mathcal{C}_{2}} \frac{\partial \mathcal{C}_{2}^{1 D}}{\partial \mathcal{C}_{1}}+\frac{\partial Y_{i}}{\partial \mathcal{C}_{3}} \frac{\partial \mathcal{C}_{3}^{1 D}}{\partial \mathcal{C}_{1}}\right)$ \\
\hline
\end{tabular}


variable Eq. (2), enthalpy Eq. (3) and mixture fraction Eq. (4).

\section{Validation results}

The tri-dimensional manifold obtained as described in Section 2 and 3 is adopted for the calculations of a bi-dimensional (in physical space) premixed laminar methane/air flame with heat loss to the wall and stratified mixture composition at the inlet, and then directly compared with detailed chemistry simulations. The geometry chosen for the calculations is a $24 \mathrm{~mm}$ wide (2D) slot burner $[1,10]$, in which the inlet is $6 \mathrm{~mm}$ wide. The inlet velocity profile is parabolic, going from a minimum of $0 \mathrm{~m} / \mathrm{s}$ at the wall to a maximum of $1 \mathrm{~m} / \mathrm{s}$ at the centerline. The walls of both burner and walls are kept at a constant temperature of $T_{w}=298 \mathrm{~K}$, and the pressure is atmospheric. The inlet temperature is $T_{u}=298 \mathrm{~K}$, and the pressure is atmospheric. The inlet mixture fraction has a parabolic profile as well, going from a lean mixture of $Z=0.033(\phi=0.6)$ at the wall to a rich mixture of $Z=0.0713(\phi=1.35)$ at the center of the inlet. The chemical reaction mechanism used for detailed calculations (as well as for the FGM) is Gri-Mech 3.0 [17], and transport coefficients are calculated by a mixture averaged approach. FGM results are directly compared with detailed chemistry simulations for validation. Detailed simulations are performed with a computational framework for combustion that has been originally developed by Groth and co-researchers ([20-22]) and modified here for the configuration under study. The equations are solved on a body-fitted, multi-block, quadrilateral mesh with adaptive mesh refinement (AMR) using a sec- ond-order accurate finite volume scheme. The refinement process is based on temperature gradients. The steady solution is obtained on a grid with a minimum size of $\Delta_{x}=0.04 \mathrm{~mm}$ covering the entire flame region. The effective grid size, calculated applying the finest resolution to the whole domain, consists of approximately 165,000 cells. FGM simulations are performed with a general purpose fluid-dynamics program [23], in which the equations are solved by means of a conservative finite-element-based control volume method. The algorithm of the software is implicit and pressure-based, and the solver chosen for the computations of this work is incompressible. The grid adopted for the FGM simulations consists of approximately 60,000 quadrilateral cells, with an adequate refinement in the entire central region. The minimum grid size in this case is $\Delta_{x}=0.06 \mathrm{~mm}$. Figure 3 shows the $\nabla \cdot\left(d_{\mathcal{C}_{k}} \nabla \mathcal{Y}\right)$ terms in the FGM results, respectively for the three control variables. A direct comparison between FGM and detailed chemistry results is shown in Fig. 4, in which the heat release profiles is displayed on the flame zone. In this figure the same iso-levels are used for detailed and FGM computations. The flame stabilizes at the dump plane, due to the combined effect of heat loss and inlet velocity profile going to zero at the wall. The flame height is represented rather well by FGM, and quite dissimilar (approximately 25\% shorter) from the $\mathrm{Le}=1$ simulations [10]. The flame is slightly shorter for the reduced model simulation, and this effect is attributed to stretch and curvature effects which become important at the flame tip and are not included in the present model. This effect is noticeable as well from the different heat release at the tip. However, the influence of the cooling walls is in very good
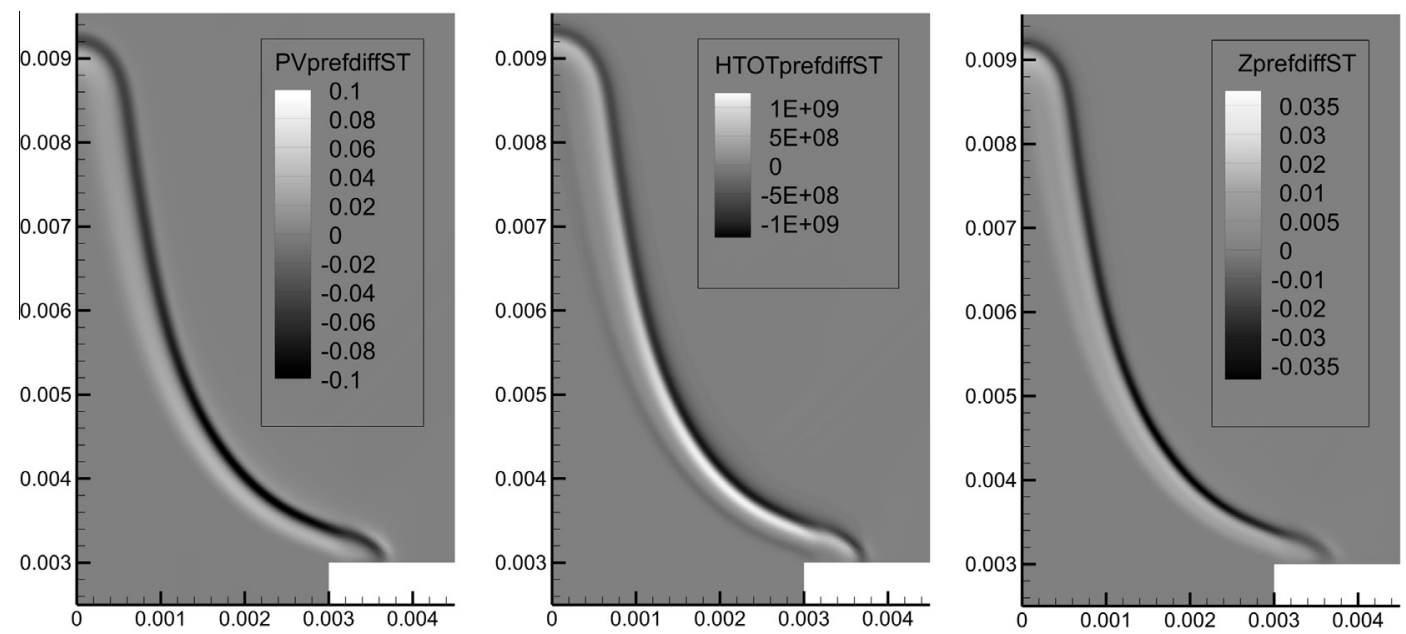

Fig. 3. Results of the FGM simulations. Magnification on the flame zone of the differential diffusion term for progress variable (top left), enthalpy (top right) and mixture fraction. The $x$ and $y$ axes are referring to spatial coordinates [m]. 


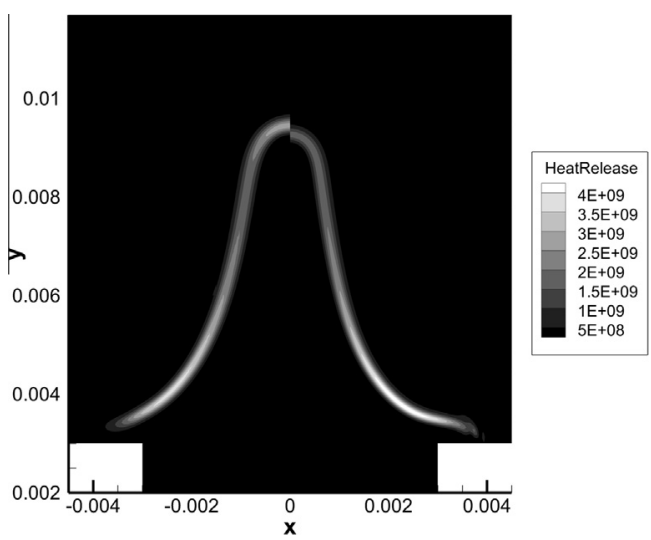

Fig. 4. Comparison between detailed chemistry (left) and FGM (right) results. Isocontours of heat release [W $\left.\mathrm{m}^{-3}\right]$. The $x$ and $y$ axes are referring to spatial coordinates $[\mathrm{m}]$.

agreement with detailed chemistry simulations. In general the FGM results of both temperature and mass fractions (not shown) demonstrate a good agreement with detailed chemistry computations from a qualitative and quantitative point of view. Besides accuracy, an important consideration about the calculation time needs to be done. The FGM simulation presented in this paper resulted in a CPU time which is approximately two orders of magnitude shorter than the detailed chemistry case. This difference is remarkable, especially considering the benefits given by the adaptive mesh refinement adopted in the detailed chemistry case. Furthermore, the calculation time and the stability of the convergence of the detailed simulations is highly affected by the flow initialization. Figure 3 describes the differential diffusion phenomenon in essence. However this is not sufficient for evaluating the differential diffusion effect on the global flame structure. Estimating the global effect of differential diffusion is in fact important in order to determine the impact (and its significance) given by its inclusion in the model. To this aim, an extra simulation is performed by using an FGM data-base computed with full transport flamelets, but omitting the differential diffusion terms in the transport equations during run-time. Thereafter, a direct comparison is performed between this simulation and the result previously given in this Section, in order to highlight the contribution of the differential diffusion terms. The simulation is performed using the same methodology, geometry, mesh, numerical methods and tabulated chemistry as formerly described. The only difference in the present case is the absence of the differential diffusion term $\nabla \cdot\left(d_{\mathcal{C}_{k}} \nabla \mathcal{Y}\right)$ in the FGM equations (i.e. the first term on the right hand side of Eq. (7)) during the calculation. The results of this simulation are shown in the left side of Fig. 5, in direct comparison with the ones of the previous section, which are given in the right side. This figure displays iso-contours of temperature, progress variable source term, heat release and $\mathrm{CO}$ mass fraction, magnified near the flame zone. The results are noticeably similar. The registered relative error on the flame height, calculated on the basis of the maximum temperature gradient, is approximately $1.5 \%$ (shorter with differential diffusion inclusion). This difference is definitely modest. This comparison is therefore indicating that differential diffusion inclusion on transport equations during the flame simulation does not provide significant benefits for methane combustion. A second important conclusion arises from this comparison: for achieving the correct flame height in FGM simulations of methane (or any other type of low-diffusive fuel) it is sufficient to produce a manifold with plain transport flamelets, but then adopt the approximated unity Lewis number FGM equations during run-time.

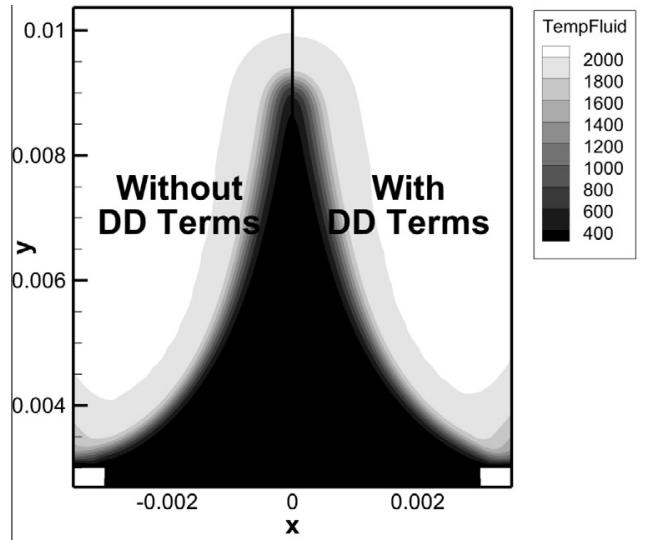

(a)

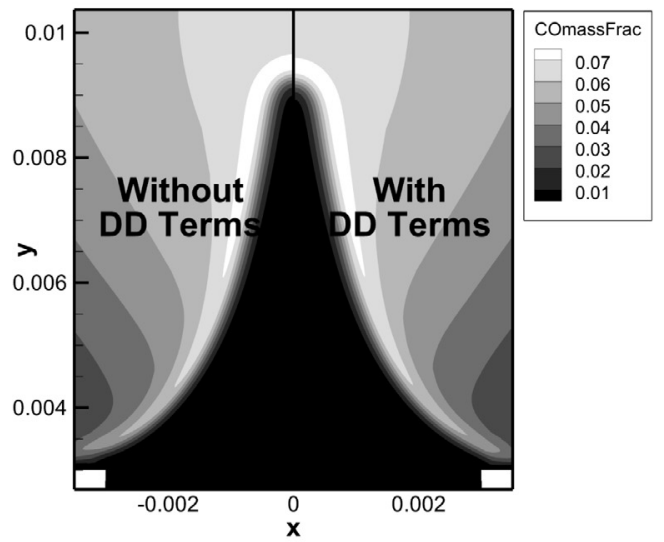

(b)

Fig. 5. Comparison between FGM results obtained with and without including the differential diffusive term: (a) temperature [K]; (b) CO mass fraction. The $x$ and $y$ axes are referring to spatial coordinates $[\mathrm{m}]$. 


\section{Conclusions}

The FGM technique is extended in order to reduce the chemistry of stratified cooled flames with the inclusion of differential diffusion effects. The combined inclusion of these effects together with differential diffusion represents an unprecedented challenge. In order to model such a system, a manifold is generated by computing flamelets with different mixture fraction and enthalpy. A set of differential diffusion coefficients are calculated and stored in the manifold. Three transport equations are solved during runtime for the progress variable, enthalpy, and mixture fraction. In order to validate the model, a bi-dimensional stratified laminar methane/air flame with heat loss to the wall and stratified inlet is computed using FGM, and subsequently compared with detailed chemistry calculations. The FGM results are in good agreement with detailed chemistry computations from a qualitative and quantitative point of view. The diffusion effects are well reproduced, albeit these are not strong in methane flames. Results indicate that differential diffusion inclusion on transport equations during the flame simulation does not provide significant benefits for methane combustion. It is also shown that for achieving the correct flame height in FGM simulations of low-diffusive fuels it is sufficient to employ a manifold constructed from plain transport flamelets, without including the extra terms of the FGM equations during run-time. Moreover, the FGM technique shows great benefits in terms of calculation time and stability of the simulation if compared to detailed chemistry. The results obtained in this paper allow to perform extensive investigations in more complex fuels, such as hydrogen, in future works.

\section{Acknowledgments}

The authors would like to express their gratitude to the Dutch Technology Foundation (STW) and Siemens Power Generation by means of the ALTAS project for the financial support.

\section{References}

[1] J.A. van Oijen, L.P.H. de Goey, Combust. Sci. Technol. 161 (2000) 113-137.

[2] O. Gicquel, N. Darabiha, D. Thévenin, Proc. Combust. Inst. 28 (2000) 1901-1908.

[3] J.A. van Oijen, L.P.H. de Goey, Combust. Theor. Model. 6 (2002) 463-478.

[4] J.A. van Oijen, L.P.H. de Goey, Combust. Theor. Model. 8 (2004) 141-163.

[5] J.A. van Oijen, R.J.M. Bastiaans, G.R.A. Groot, L.P.H. de Goey, Proc. Combust. Inst. 30 (2005) 657-664.

[6] A.W. Vreman, B.A. Albrecht, J.A. van Oijen, L.P.H. de Goey, R.J.M. Bastiaans, Combust. Flame 153 (2008) 394-416.

[7] W.J.S. Ramaekers, J.A. van Oijen, L.P.H. de Goey, Combust. Theor. Model. 16 (2012) 943-975.

[8] A. Donini, S.M. Martin, R.J.M. Bastiaans, L.P.H. de Goey, Proceedings of the ASME Turbo Expo, ASME, 2013, GT2013-94363.

[9] M. Matalon, Annu. Rev. Fluid Mech. 39 (2007) 163191.

[10] A. Donini, R.J.M. Bastiaans, L.P.H. de Goey, Proceedings of the ASME Turbo Expo, ASME, 2014, GT2014-26210.

[11] J.A.M. de Swart, R.J.M. Bastiaans, J.A. van Oijen, L.P.H. de Goey, R.S. Cant, Flow Turbul. Combust. 85 (2010) 473-511.

[12] G. Steinhilber, U. Maas, Proc. Combust. Inst. 34 (2013) 217-224.

[13] P.H. de Almeida Konzen, T. Richter, U. Riedel, U. Maas, Combust. Theor. Model. 15 (2011) 299-323.

[14] R.W. Bilger, Combust. Flame 80 (1990) 135-149.

[15] H. Pitsch, H. Steiner, Phys. Fluids 12 (2000) 25412554.

[16] Chem1D, One-dimensional Laminar Flame Code, $<$ http://www.combustion.tue.nl/chemld $>$.

[17] G.P. Smith, D.M. Golden, M. Frenklach, et al., Gri-Mech 3.0.

[18] V. Hiremath, S.R. Lantz, H. Wang, S.B. Pope, Combust. Flame 159 (2012) 3096-3109.

[19] M. Ihme, C. Schmitt, H. Pitsch, Proc. Combust. Inst. 32 (2009) 1527-1535.

[20] C.P.T. Groth, S.A. Nothrup, AIAA (2005) 20055333.

[21] M.R. Charest, C.P.T. Groth, Ö. L Gülder, Combust. Theor. Model. 14 (2010) 793-825.

[22] F.E. Hernández Pérez, F.T.C. Yuen, C.P.T. Groth, Ö.L. Gülder, Proc. Combust. Inst. 33 (2011) 13651371.

[23] Ansys-CFX 13.0, A High-Performance, General Purpose Fluid Dynamics Program, 2012. 